\title{
FROM PROJECTIVE REPRESENTATIONS TO QUASI-QUANTUM GROUPS
}

\author{
HUA-LIN HUANG
}

\begin{abstract}
This is a contribution to the project of quiver approaches to quasi-quantum groups initiated in [13]. We classify Majid bimodules over groups with 3-cocycles by virtue of projective representations. This leads to a theoretic classification of graded pointed Majid algebras over path coalgebras, or equivalently cofree pointed coalgebras, and helps to provide a projective representation-theoretic description of the gauge equivalence of graded pointed Majid algebras. We apply this machinery to construct some concrete examples and obtain a classification of finitedimensional graded pointed Majid algebras with the set of group-likes equal to the cyclic group $\mathbb{Z}_{2}$ of order 2 .
\end{abstract}

Keywords Majid algebra, projective representation, Hopf quiver 2000 MR Subject Classification 16W30, 16W35, 20C25

\section{INTRODUCTION}

Quasi-quantum groups, that is quasi-Hopf algebras, were introduced by Drinfeld [7] in accordance with his philosophy of quantum groups. Since its appearance, the notion has been playing important roles in various areas of mathematics and physics. Our goal is to classify some interesting classes of quasi-quantum groups and their representations. Since the classification problem of general quasi-quantum groups is still very complicated, we focus on elementary (i.e., finite-dimensional and the simple modules of the underlying algebras are 1-dimensional) quasi-Hopf algebras and pointed (i.e., the simple comodules of the underlying coalgebras are 1-dimensional) Majid algebras (=dual quasi-Hopf algebras). In this situation we can take advantage of the well-developed representation theory of algebras (see for instance [1]), especially the quiver techniques.

A quiver setting for quasi-quantum groups is provided in [13]. It is shown that a systematic study of elementary quasi-Hopf algebras or pointed Majid algebras, in particular the classification problem and the associated representation theory, can be carried out effectively in this framework by virtue of the quiver techniques. Under our quiver setting the study of elementary quasi-Hopf algebras can be included in that of pointed Majid algebras, so 
we always work on the latter only. The present paper is devoted to this quiver classification project of pointed Majid algebras.

For an arbitrary pointed Majid algebra, one can always associate to it a coradically graded version induced by its coradical filtration. So in this paper, by graded we actually mean coradically graded. According to a Gabriel type theorem for pointed Majid algebras [13], any graded pointed Majid algebra can be embedded into a graded Majid algebra structure on some unique Hopf quiver. Therefore the first step of the classification project is to investigate the graded Majid algebra structures on Hopf quivers. The aim of this paper is to give an explicit classification of such Majid algebras.

Let $k$ be a field. For a Hopf quiver $Q$, a graded Majid algebra structure on the path coalgebra $k Q$ determines on its set of vertices $Q_{0}$ a group structure $G$ with a 3 -cocycle $\Phi$ and a $(k G, \Phi)$-Majid bimodule structure on the space $k Q_{1}$ spaned by the set of arrows. Conversely, given a group $G$ with a $3-$ cocycle $\Phi$ and a $(k G, \Phi)$-Majid bimodule $M$, we can associate to the data a Hopf quiver $Q$ and a graded Majid algebra structure on the path coalgebra $k Q$. See [13] for the precise description. So for our purpose, it suffices to classify all $(k G, \Phi)$-Majid bimodules for a general group $G$ and an arbitrary 3-cocycle $\Phi$.

The notion of Majid bimodules is certainly an analog of the familiar one of Hopf bimodules. Nichols [20] introduced the definition of Hopf bimodules and initiated the classification over finite abelian groups. In [4, 5], Cibils and Rosso gave an explicit classification of the Hopf bimodules over an arbitrary group and applied the result to define Hopf quivers and provide the complete classification of graded Hopf structures on path (co)algebras. In more detail, for a group $G$, the category of $k G$-Hopf bimodules is equivalent to the product of the categories of usual module categories $\prod_{C \in \mathcal{C}} k Z_{C}-\bmod$, where $\mathcal{C}$ is the set of conjugacy classes and $Z_{C}$ is the centralizer of one of the elements in the class $C \in \mathcal{C}$; and via the quantum shuffle product [21] on Hopf quivers determined by these data of $k G$-Hopf bimodules, a complete list of graded cofree pointed Hopf algebras is obtained. Here by "cofree" we mean the underlying coalgebras are cotensor coalgebras and therefore they enjoy the universal mapping property (see [20]).

Our main result is an extension of Cibils and Rosso's works to Majid bimodules and Majid algebras. It turns out that the category of $(k G, \Phi)$-Majid bimodules is equivalent to the product of categories $\prod_{C \in \mathcal{C}}\left(k Z_{C}, \tilde{\Phi}_{C}\right)$-rep, where $\mathcal{C}, Z_{C}$ are as before and $\tilde{\Phi}_{C}$ is a 2-cocycle on $Z_{C}$ determined by $\Phi$ and $\left(k Z_{C}, \tilde{\Phi}_{C}\right)$-rep is the category of projective $\tilde{\Phi}_{C}$-representations in the sense of Schur, or equivalently the left module category of the twisted group algebra $k^{\tilde{\Phi}_{C}} Z_{C}$ (see for example[15]). As applications of this result, we obtain a 
complete classification of graded Majid algebra structures on the path coalgebras of Hopf quivers, which amounts to a classification of graded cofree pointed Majid algebras, and a projective representation-theoretic interpretation of their gauge equivalence. We apply this machinery to construct some concrete examples of Majid bimodules and Majid algebras, in which the group $G$ is the cyclic group $\mathbb{Z}_{2}$ of order 2 . A classification of finitedimensional graded pointed Majid algebras over $\mathbb{Z}_{2}$ is obtained by making use of the Gabriel type theorem.

Recently, Etingof and Gelaki have a series of works [8, 9, 10, 11] on the classification of elementary quasi-Hopf algebras over the field of complex numbers. By the well-known Tannakian formalism, their works lead to some interesting classification results for finite tensor categories (see [2]). Their classification method bases on very clever constructions "by hand", which is quite different from ours. We will show that our approach can provide a conceptual understanding for their direct constructions by the projective representation theory of groups.

The layout of the paper is as follows. In Section 2 we give the definitions of Majid modules and Majid bimodules and include a fundamental structure result for Majid modules. In Section 3 we provide the explicit classification of Majid bimodules over groups with 3-cocycles. In Section 4 we give, via the classification result, a theoretic classification of graded Majid algebra structures over path coalgebras and a description of their gauge equivalence. In Section 5 we provide some concrete examples and obtain a classification of finite-dimensional graded pointed Majid algebras over $\mathbb{Z}_{2}$.

Throughout, we fix a ground field $k$ and vector spaces, linear mappings, (co)algebras and unadorned tensor product $\otimes$ are over $k$. Unexplained notions about Hopf algebras, quasi-Hopf algebras and Majid algebras can be found in the books $[16,19,25]$. We turn to [13] frequently for notations and results of the quiver setting of Majid algebras.

\section{Majid Modules and Fundamental Structure}

We recall the notion of Majid bimodules for the convenience of the reader. For our purpose we also include the notion of Majid modules and an analog of Larson and Sweedler's Fundamental Theorem for Hopf modules [17].

Definition 2.1. Assume that $H$ is a Majid algebra with reassociator $\Phi$. A linear space $M$ is called an $H$-Majid bimodule, if $M$ is an $H$-bicomodule with structure maps $\left(\delta_{L}, \delta_{R}\right)$, and there are two $H$-bicomodule morphisms

$$
\rho_{L}: H \otimes M \longrightarrow M, h \otimes m \mapsto h . m, \quad \rho_{R}: M \otimes H \longrightarrow M, m \otimes h \mapsto m . h
$$


such that for all $g, h \in H, m \in M$, the following equalities hold:

$$
\begin{gathered}
1_{H} \cdot m=m=m \cdot 1_{H}, \\
g_{1} \cdot\left(h_{1} \cdot m_{0}\right) \Phi\left(g_{2}, h_{2}, m_{1}\right)=\Phi\left(g_{1}, h_{1}, m^{-1}\right)\left(g_{2} h_{2}\right) \cdot m^{0}, \\
m_{0} \cdot\left(g_{1} h_{1}\right) \Phi\left(m_{1}, g_{2}, h_{2}\right)=\Phi\left(m^{-1}, g_{1}, h_{1}\right)\left(m^{0} \cdot g_{2}\right) \cdot h_{2}, \\
g_{1} \cdot\left(m_{0} \cdot h_{1}\right) \Phi\left(g_{2}, m_{1}, h_{2}\right)=\Phi\left(g_{1}, m^{-1}, h_{1}\right)\left(g_{2} \cdot m^{0}\right) \cdot h_{2},
\end{gathered}
$$

where we use the Sweedler notation

$$
\Delta(g)=g_{1} \otimes g_{2}, \quad \delta_{L}(m)=m^{-1} \otimes m^{0}, \quad \delta_{R}(m)=m_{0} \otimes m_{1}
$$

for coproduct and comodule structure maps.

Suppose only the $H$-bicomodule morphism $\rho_{L}$ is defined for the $H$-bicomodule $M$ satisfying $1_{H} . m=m$ and (2.2), we call it a left $H$-Majid module. Similarly we define a right $H$-Majid module.

For the purpose of the present paper, it is enough to focus on the case of $H$ being a group algebra with 3 -cocycle. This also allows better exposition without much loss of generality.

From now on, we fix a group $G$ with unit element $\epsilon$ and a 3-cocycle $\Phi: G \times G \times G \longrightarrow k^{*}$. Recall that, $\Phi$ being a 3 -cocycle means the following equalities hold for all $e, f, g, h \in G$ :

$$
\begin{gathered}
\Phi(e, f, g) \Phi(e, f g, h) \Phi(f, g, h)=\Phi(e f, g, h) \Phi(e, f, g h), \\
\Phi(\epsilon, e, f)=\Phi(e, \epsilon, f)=\Phi(e, f, \epsilon)=1 .
\end{gathered}
$$

In the literature, such a cocycle is said to be normalized. All the cocycles in this paper are assumed so. We extend $\Phi$, without changing the notation, by linearity to a function on $(k G)^{\otimes 3}$ and understand the group algebra $k G$ as a Majid algebra with reassociator $\Phi$.

Let $M$ be a $(k G, \Phi)$-Majid bimodule. Then in the first place the underlying bicomodule structure makes it a $G$-bigraded space $M=\bigoplus_{g, h \in G}{ }^{g} M^{h}$ with $(g, h)$-isotypic component

$$
{ }^{g} M^{h}=\left\{m \in M \mid \delta_{L}(m)=g \otimes m, \delta_{R}(m)=m \otimes h\right\} .
$$

The quasi-bimodule structure maps satisfy the following equalities:

$$
\begin{aligned}
e .(f . m) & =\frac{\Phi(e, f, g)}{\Phi(e, f, h)}(e f) . m, \\
(m . e) . f & =\frac{\Phi(h, e, f)}{\Phi(g, e, f)} m .(e f), \\
(e . m) . f & =\frac{\Phi(e, h, f)}{\Phi(e, g, f)} e .(m . f),
\end{aligned}
$$

for all $e, f, g, h \in G$ and $m \in{ }^{g} M^{h}$. 
For the classification of Majid bimodules, first we should study Majid modules and develop an analog of the fundamental structure theorem for Hopf modules. A right $(k G, \Phi)$-Majid module is a $k G$-bicomodule $M$ with a right quasi-module satisfying (2.8). A trivial example of right $(k G, \Phi)$-Majid module is $k G$ with bicomodule defined by the usual diagonal map and right quasi-module by the right multiplication. It is also easy to see that for any left $k G$-comodule $\left(V, d_{L}\right)$ we can define a right $(k G, \Phi)$-Majid module on the tensor space $V \otimes k G$, with structure maps given by

$$
\begin{gathered}
\delta_{L}(v \otimes g)=h g \otimes v \otimes g, \quad \delta_{R}(v \otimes g)=v \otimes g \otimes g, \\
(v \otimes g) . f=\Phi^{-1}(h, g, f) v \otimes g f,
\end{gathered}
$$

for all $f, g, h \in G$ and $v \in{ }^{h} V=\left\{v \in V \mid d_{L}(v)=h \otimes v\right\}$. For any right $k G$-comodule $V$, a left $(k G, \Phi)$-Majid module on $k G \otimes V$ can be defined analogously. Majid modules of this form are said to be trivial.

The fundamental structure of Majid modules is described in the following.

Proposition 2.2. The category $\mathcal{R} \mathcal{M}(k G, \Phi)$ of right $(k G, \Phi)$-Majid modules and the category $k G$-comod of left $k G$-comodules are equivalent. In particular, right $(k G, \Phi)$-Majid modules are trivial.

Proof. For each $M \in \mathcal{R} \mathcal{M}(k G, \Phi)$, let $M^{\epsilon}=\left\{m \in M \mid \delta_{R}(m)=m \otimes \epsilon\right\}$ be the space of right coinvariants. It is a sub left $k G$-comodule of $M$. Define a functor $\Theta: \mathcal{R} \mathcal{M}(k G, \Phi) \longrightarrow k G-\operatorname{comod}$ by $M \mapsto M^{\epsilon}$.

For each $V \in k G$-comod, we consider the right $(k G, \Phi)$-Majid module structure on $V \otimes k G$ as given by (2.10)-(2.11). Define a functor $\Xi$ : $k G-\operatorname{comod} \longrightarrow \mathcal{R} \mathcal{M}(k G, \Phi)$ by $V \mapsto V \otimes k G$.

The verification of these functors actually providing the claimed equivalence of categories is routine. We only mention the isomorphism maps of $M$ and $M^{\epsilon} \otimes k G$ in which the 3-cocycle $\Phi$ must get involved. Define $\zeta: M \longrightarrow M^{\epsilon} \otimes k G$ via $m \mapsto \frac{\Phi\left(e, f^{-1}, f\right)}{\Phi\left(f, f^{-1}, f\right)} m . f^{-1} \otimes f$ for all $m \in{ }^{e} M^{f}$. For the converse, define $\xi: M^{\epsilon} \otimes k G \longrightarrow M$ via $v \otimes g \mapsto v . g$.

Remark 2.3. If we consider also the monoidal structures on the categories $\mathcal{R} \mathcal{M}(k G, \Phi)$ and $k G$-comod, then the previous equivalence is actually monoidal. The result can be generalized to Majid modules over general Majid algebras with proper modification. The quasi-Hopf version of the fundamental structure theorem of Hopf modules is developed in [12].

\section{Majid Bimodules over Groups}

Denote the category of $(k G, \Phi)$-Majid bimodules by $\mathcal{M B}(k G, \Phi)$. For each $M \in \mathcal{M B}(k G, \Phi)$, let $M=\bigoplus_{g, h \in G}{ }^{g} M^{h}$ be the decomposition of isotypic 
components. According to the axioms of $(k G, \Phi)$-Majid bimodules, we have for all $f, g, h \in G$,

$$
f .{ }^{g} M^{h}={ }^{f g} M^{f h}, \quad{ }^{g} M^{h} . f={ }^{g f} M^{h f} .
$$

In particular it follows that for all $x, g, c \in G$,

$$
{ }^{g^{-1}} \operatorname{cgx} M^{x}={ }^{-1} c g M^{\epsilon} \cdot x=\left(\left(g^{-1} \cdot{ }^{c} M^{\epsilon}\right) \cdot g\right) \cdot x .
$$

It is clear that

$$
\operatorname{dim}_{k}{ }^{-1} \operatorname{cgx} M^{x}=\operatorname{dim}_{k}^{c} M^{\epsilon} .
$$

Now we are ready to classify Majid bimodules over groups. Firstly, by Proposition 2.2, we have $M \cong M^{\epsilon} \otimes k G$ as right $(k G, \Phi)$-Majid modules. The space $M^{\epsilon}$ of right coinvariants is a sub left comodule, but not closed under the left quasi-module action $\rho_{L}$. However, if we consider the conjugate action, then by (3.2) we have $(g \cdot v) \cdot g^{-1} \in M^{\epsilon}$ for all $v \in M^{\epsilon}$ and $g \in G$. For convenience, we use the notation $g \triangleright v=(g \cdot v) \cdot g^{-1}$. Of course, in general this action does not make a usual $G$-representation. It turns out to be quite similar to Schur's projective representation of $G$. More precisely, for all $e, f, g \in G, v \in{ }^{g} M^{\epsilon}$, we have

$$
\epsilon \triangleright v=v, \quad e \triangleright(f \triangleright v)=\tilde{\Phi}(e, f, g)(e f) \triangleright v,
$$

where $\tilde{\Phi}(e, f, g)=\frac{\Phi(e, f, g) \Phi\left(e f, f^{-1}, e^{-1}\right) \Phi\left(e, f g, f^{-1}\right)}{\Phi\left(e f g, f^{-1}, e^{-1}\right) \Phi\left(e, f, f^{-1}\right)}$. For each $g$, we choose for ${ }^{g} M^{\epsilon}$ a basis $\left\{v_{g}(\lambda)\right\}_{\lambda \in \Lambda_{g}}$, then construct and index a basis $\left\{v_{g}(\lambda)\right\}_{g \in G, \lambda \in \Lambda_{g}}$ for $M^{\epsilon}$ accordingly. Let $X: G \longrightarrow G L\left(M^{\epsilon}\right)$ be the associated matrix representation of the action $\triangleright$ under this basis. Then we have for all $e, f \in G$

$$
X(\epsilon)=\operatorname{Id}_{M^{\epsilon}}, \quad X(e) X(f)=\chi(e, f) X(e f),
$$

where $\chi(e, f)$ is a diagonal matrix, with scalar block $\tilde{\Phi}\left(e, f, f^{-1} e^{-1} g e f\right) \operatorname{Id} g_{M^{\epsilon}}$ corresponding to the subset $\left\{v_{g}(\lambda)\right\}_{\lambda \in \Lambda_{g}}$ of basis elements of $M^{\epsilon}$. Recall that a mapping $\rho: G \longrightarrow G L(V)$ is called a projective representation of $G$ if there exists a mapping $\alpha: G \times G \longrightarrow k^{*}$ such that $\rho(\epsilon)=\operatorname{Id}_{V}$ and $\rho(g) \rho(h)=\alpha(g, h) \rho(g h)$ for all $g, h \in G$. In the last equality $\alpha(g, h)$ is understood as a scalar matrix. The representation is also called an $\alpha$ representation when we need to specify the mapping. It is clear that $\alpha$ is a 2-cocycle on the group $G$. If the 2 -cocycle $\alpha$ is trivial, that is $\alpha(g, h)=1$ for all $g, h \in G$, then of course $\rho$ is a usual linear representation. In the just defined mapping $X: G \longrightarrow G L\left(M^{\epsilon}\right)$ the $\chi(e, f)$ 's are diagonal matrices consisting of different scalar blocks, thus in general $X$ is not a projective representation. However, the scalar blocks vary regularly via the function $\tilde{\Phi}$ determined by the 3 -cocycle $\Phi$, so we might call $X$ a $\Phi$-twisted projective 
representation, which seems to be a natural generalization of the projective representation.

Here it is worthy to digress for a moment. Note that for $M^{\epsilon}$ the compatibility of the conjugate action $\triangleright$ and the left coaction becomes

$$
\delta_{L}(f \triangleright v)=f g f^{-1} \otimes f \triangleright v
$$

for all $f, g \in G, v \in{ }^{g} M^{\epsilon}$. The data $\left(M^{\epsilon}, \triangleright, \delta_{L}\right)$ satisfying (3.6) can be viewed as the right candidate of crossed module in the sense of [26], or the more commonly used term Yetter-Drinfeld module, for Majid algebras. We call the triple $\left(M^{\epsilon}, \triangleright, \delta_{L}\right)$ a left twisted crossed $(k G, \Phi)$-module and it is also how $M^{\epsilon}$ inherits the left $(k G, \Phi)$-Majid module structure of $M$.

We proceed to further investigation of $M^{\epsilon}$ via the twisted projective representation $X$. Let $\mathcal{C}$ be the set of the conjugacy classes of $G$. For each $C \in \mathcal{C}$, let ${ }^{C} M^{\epsilon}=\oplus_{g \in C}{ }^{g} M^{\epsilon}$. Again by (3.2), the action $\triangleright$ is closed for each ${ }^{C} M^{\epsilon}$, so we have the direct sum decomposition of $M$ into sub twisted projective representations, and even sub twisted crossed modules:

$$
M=\bigoplus_{C \in \mathcal{C}}{ }^{C} M^{\epsilon}
$$

Moreover, for each $g \in G$, let $Z_{g}$ be its centralizer, then we can restrict the action $\triangleright$ to $Z_{g}$ on ${ }^{g} M^{\epsilon}$. Let $X_{g}: Z_{g} \longrightarrow G L\left({ }^{g} M^{\epsilon}\right)$ denote the corresponding matrix representation. The following observation is a key step to our classification result.

Lemma 3.1. $X_{g}$ is a projective representation of $Z_{g}$ for all $g \in G$.

Proof. By (3.5) we have $X_{g}(\epsilon)=\operatorname{Id}{ }_{g_{M}}$ and $X_{g}(e) X_{g}(f)=\tilde{\Phi}(e, f, g) X_{g}(e f)$ for all $e, f \in Z_{g}$. Denote by

$$
\tilde{\Phi}_{g}: Z_{g} \times Z_{g} \longrightarrow k^{*}, \quad(e, f) \mapsto \tilde{\Phi}(e, f, g)
$$

the mapping induced by $\tilde{\Phi}$. Direct calculation indicates that $\tilde{\Phi}_{g}$ is a 2-cocycle on $Z_{g}$, thus $X_{g}$ is a projective representation of $Z_{g}$.

Since ${ }^{C} M^{\epsilon}=\oplus_{g \in C}{ }^{g} M^{\epsilon}$, so it is of interest to view the twisted projective representation ${ }^{C} M^{\epsilon}$ of $G$ as the gluing of the set of the usual projective representations $\left\{{ }^{g} M^{\epsilon} \mid g \in C\right\}$ of the centralizers $Z_{g}$.

For each $g \in G$, let $C_{g}$ be the conjugacy class containing $g$. Just as the classical group representation theory, we want to extend the $\tilde{\Phi}_{g^{-}}$-representation ${ }^{g} M^{\epsilon}$ of $Z_{g}$ to the twisted projective representation ${ }^{C_{g}} M^{\epsilon}$ of $G$ by an appropriate induction procedure. To this end, we consider a twisted version 
of tensor product of $k G$, viewed as a right $Z_{g^{-}}$representation, with the $\tilde{\Phi}_{g^{-}}$ representation ${ }^{g} M^{\epsilon}$ of $Z_{g}$, as the quotient space

$$
\frac{k G \otimes{ }_{k}{ }^{g} M^{\epsilon}}{<e \otimes f \triangleright m-\tilde{\Phi}(e, f, g) e f \otimes m \mid \forall e \in G, f \in Z_{g}, m \in{ }^{g} M^{\epsilon}>} .
$$

We denote it by $k G \hat{\otimes}_{k Z_{g}}{ }^{g} M^{\epsilon}$. For each $e \otimes m \in k G \otimes_{k}{ }^{g} M^{\epsilon}$, let $e \hat{\otimes} m$ be its image in $k G \hat{\otimes}_{k Z_{g}}{ }^{g} M^{\epsilon}$. Define two linear mappings as follows:

$$
\begin{aligned}
d_{L}: k G \hat{\otimes}_{k Z_{g}}{ }^{g} M^{\epsilon} & \longrightarrow k G \otimes k G \hat{\otimes}_{k Z_{g}}{ }^{g} M^{\epsilon}, \\
e \hat{\otimes} m & \mapsto e g e^{-1} \otimes e \hat{\otimes} m, \\
m_{L}: k G \otimes k G \hat{\otimes}_{k Z_{g}}{ }^{g} M^{\epsilon} & \longrightarrow k G \hat{\otimes}_{k Z_{g}}{ }^{g} M^{\epsilon}, \\
f \otimes e \hat{\otimes} m & \mapsto \tilde{\Phi}(f, e, g) f e \hat{\otimes} m .
\end{aligned}
$$

By direct verification one can show that

Lemma 3.2. The triple $\left(k G \hat{\otimes}_{k Z_{g}}{ }^{g} M^{\epsilon}, m_{L}, d_{L}\right)$ makes a twisted crossed module for $(k G, \Phi)$ and we have the following canonical isomorphism

$$
{ }^{C_{g}} M^{\epsilon} \cong k G \hat{\otimes}_{k Z_{g}}{ }^{g} M^{\epsilon} .
$$

We are now in a position to state the main result. First we abbreviate some notations. For each $C \in \mathcal{C}$, let $Z_{C}$ denote the centralizer of one element in $C$, say $g(C)$, and $\tilde{\Phi}_{C}$ the corresponding 2-cocycle $\tilde{\Phi}_{g(C)}$ on $\mathbb{Z}_{C}$ as defined in (3.8), and $M_{C}$ the $\tilde{\Phi}_{C}$-representation ${ }^{g(C)} M^{\epsilon}$ of $Z_{C}$. Let $\left(k Z_{C}, \tilde{\Phi}_{C}\right)$-rep denote the category of $\tilde{\Phi}_{C}$-representations of $Z_{C}$ and $\prod_{C \in \mathcal{C}}\left(k Z_{C}, \tilde{\Phi}_{C}\right)$-rep their cartesian product. By $k^{\tilde{\Phi}_{C}} Z_{C}$ we denote the twisted group algebra and by $k^{\tilde{\Phi}_{C}} Z_{C}-$ mod we denote its left module category. Let $\prod_{C \in \mathcal{C}} k^{\tilde{\Phi}_{C}} Z_{C}-\bmod$ denote the cartesian product of categories.

Theorem 3.3. We have the following equivalence of categories:

$$
\mathcal{M B}(k G, \Phi) \cong \prod_{C \in \mathcal{C}}\left(k Z_{C}, \tilde{\Phi}_{C}\right)-\mathrm{rep} \cong \prod_{C \in \mathcal{C}} k^{\tilde{\Phi}_{C}} Z_{C}-\bmod .
$$

Proof. The latter equivalence is known (see [15]). We prove the former one. Define a functor

$$
\Theta: \mathcal{M B}(k G, \Phi) \longrightarrow \prod_{C \in \mathcal{C}}\left(k Z_{C}, \tilde{\Phi}_{C}\right)-\text { rep }
$$

by $M \mapsto\left(M_{C}\right)_{C \in \mathcal{C}}$. For the converse, define a functor

$$
\Xi: \prod_{C \in \mathcal{C}}\left(k Z_{C}, \tilde{\Phi}_{C}\right)-\operatorname{rep} \longrightarrow \mathcal{M B}(k G, \Phi)
$$

by $\left(V_{C}\right)_{C \in \mathcal{C}} \mapsto \bigoplus_{C \in \mathcal{C}} k G \hat{\otimes}_{k Z_{C}} V_{C} \otimes k G$. The $(k G, \Phi)$-Majid bimodule structure on the latter is furnished by (3.9)-(3.10) and (2.10)-(2.11). Straightforward but tedious calculation shows that the functors $\Theta$ and $\Xi$ provide the desired category equivalence. 
We should note that the collections of projective representations which give rise to Majid bimodules are special, as the occurred 2-cocycles are uniformly determined by a 3-cocycle. For later application, these collections are said to be admissible. When the 3-cocylcles need to be emphasized, those corresponding to $(k G, \Phi)$-Majid bimodules are called $\Phi$-collections.

We remark that, by the previous category equivalence, it is easy to derive some interesting consequences in the case of $G$ being a finite group. By Maschke's theorem and its generalization, the category $\mathcal{M B}(k G, \Phi)$ is semisimple if and only if the character of the field $k$ does not divide the order of $G$. When $k$ is algebraically closed with characteristic 0 , the set of simple $(k G, \Phi)$-Majid bimodules is in one-to-one correspondence with the set of simple $k^{\tilde{\Phi}_{C}} Z_{C}$-modules, which in turn is in one-to-one correspondence with the $\tilde{\Phi}_{C}$-regular conjugacy classes of $Z_{C}$ for all $C \in \mathcal{C}$ (see [15]). We would also like to remark that the category $\mathcal{M B}(k G, \Phi)$ can be described by the module category of the twisted quantum double $D^{\Phi}(G)$ introduced in [6]. This is done by generalizing the corresponding results for Hopf bimodules in $[21,23]$ to the Majid setting. The method adopted in this paper is more elementary and straightforward, since the representation theory of a group is much easier than that of the complicated twisted quantum double.

\section{Quiver Majid Algebras and Gauge Equivalence}

As applications of our main result, in this section we give a classification of the graded Majid algebras on the path coalgebras of Hopf quivers and a description for the gauge equivalence.

Fix a group $G$ and a 3 -cocycle $\Phi$. Let $\mathcal{S}(g)=g^{-1}, \alpha(g)=1$ and $\beta(g)=$ $1 / \Phi\left(g, g^{-1}, g\right)$ for all $g \in G$. We understand $(k G, \Phi)$ as a Majid algebra with quasi-antipode $(\mathcal{S}, \alpha, \beta)$. A ramification datum of the group $G$ is a formal sum $R=\sum_{C \in \mathcal{C}} R_{C} C$ of conjugacy classes with non-negative integer coefficients. Let $Q(G, R)$ denote the corresponding Hopf quiver (see [5]): the set of vertices is $G$, and for each $x \in G$ and $c \in C$, there are $R_{C}$ arrows going from $x$ to $c x$. It is proved in [13] that

Proposition 4.1. Let $G$ be a group, $\Phi$ a 3-cocycle, $R$ a ramification datum, and $Q=Q(G, R)$ the associated Hopf quiver. Then the path coalgebra $k Q$ admits a graded Majid algebra structure with $k Q_{0} \cong(k G, \Phi, \mathcal{S}, \alpha, \beta)$ as a sub Majid algebra if and only if $k Q_{1}$ admits a $(k G, \Phi)$-Majid bimodule structure. Moreover, the set of such graded Majid algebra structures on the path coalgebra $k Q$ is in one-to-one correspondence with the set of $(k G, \Phi)$-Majid bimodule structures on $k Q_{1}$.

As a corollary of Theorem 3.3 and Proposition 4.1, we have 
Theorem 4.2. Let $G$ be a group, $\Phi$ a 3-cocycle, $R=\sum_{C \in \mathcal{C}} R_{C} C$ a ramification datum, and $Q=Q(G, R)$ the associated Hopf quiver. Then the set of graded Majid algebra structures on the path coalgebra $k Q$ with $k Q_{0} \cong$ $(k G, \Phi, \mathcal{S}, \alpha, \beta)$ as a sub Majid algebra is in one-to-one correspondence with the set of collections $\left(M_{C}\right)_{C \in \mathcal{C}}$ where $M_{C}$ is a $\tilde{\Phi}_{C}$-representation of $Z_{C}$ of dimension $R_{C}$.

Note that we do not lose much of the generality by fixing the quasiantipode of $(k G, \Phi)$ as $(\mathcal{S}, \alpha, \beta)$ defined above, since on the one hand the quasi-antipodes have nothing to do with the Majid bimodules; on the other hand by [7] an arbitrary quasi-antipode is of the form

$$
\left(\mu * \mathcal{S} * \mu^{-1}, \quad \mu * \alpha, \quad \beta * \mu^{-1}\right)
$$

where $*$ is the convolution product and $\mu \in \operatorname{Hom}_{k}(k G, k)$ is a convolution invertible function. In addition, cofree pointed coalgebras can always be presented as path coalgebras (see [5]), so the preceding theorem amounts to a complete classification of graded cofree pointed Majid algebras.

In the rest of this section, we study the gauge equivalence of graded pointed Majid algebras. First we recall the definition. Let $(H, \Phi, \mathcal{S}, \alpha, \beta)$ be a Majid algebra. A gauge transformation on $H$ is a convolution-invertible 2-cochain $F: H \otimes H \longrightarrow k$ obeying $F\left(h, 1_{H}\right)=F\left(1_{H}, h\right)=\varepsilon(h)$ for all $h \in H$. Given a gauge transformation $F$ on $H$, there is a new Majid algebra $H_{F}$ with new product, $\Phi, \alpha, \beta$ given by

$$
\begin{gathered}
g \cdot_{F} h=F^{-1}\left(g_{1}, h_{1}\right) g_{2} \cdot h_{2} F\left(g_{3}, h_{3}\right), \\
\Phi_{F}(f, g, h)= \\
F^{-1}\left(g_{1}, h_{1}\right) F^{-1}\left(f_{1}, g_{2} \cdot h_{2}\right) \Phi\left(f_{2}, g_{3}, h_{3}\right) F\left(f_{3} \cdot g_{4}, h_{4}\right) F\left(f_{4}, g_{5}\right), \\
\alpha_{F}(h)=F\left(\mathcal{S}\left(h_{1}\right), h_{3}\right) \alpha\left(h_{2}\right), \quad \beta_{F}(h)=F^{-1}\left(h_{1}, \mathcal{S}\left(h_{3}\right)\right) \alpha\left(h_{2}\right)
\end{gathered}
$$

for all $f, g, h \in H$. Two Majid algebras $H$ and $H^{\prime}$ are said to be gauge equivalent if there exists a gauge transformation $F$ on $H$ such that $H_{F}$ and $H$ are isomorphic Majid algebras. The gauge equivalence is an equivalence relation.

We introduce a gauge equivalence on Majid bimodules over a group $G$ and use it to describe the gauge equivalence of the associated Majid algebras. If a 2-cochain $F: G \times G \longrightarrow k^{*}$ is convolution-invertible and satisfies $F(g, \epsilon)=$ $F(\epsilon, g)=1$ for all $g \in G$, we call it a gauge transformation on $G$. Define $\Phi_{F}: G \times G \times G \longrightarrow k^{*}$ by

$$
\Phi_{F}(e, f, g)=\frac{F(e, f) F(e f, g)}{F(e, f g) F(f, g)} \Phi(e, f, g) .
$$


Clearly $\Phi_{F}=\Phi \partial F$, where $\partial F$ is the differential of $F$ and the product is the convolution product. So it is a 3 -cocycle and cohomologous to $\Phi$. Given a $(k G, \Phi)$-Majid bimodule $M$ and a gauge transformation $F$ on $G$, we can build a $\left(k G, \Phi_{F}\right)$-Majid bimodule $M_{F}$ as follows. The space and the $k G$ bicomodule are the same as $M$, while the new quasi-bimodule structure maps are given by

$$
f_{\cdot F} m=\frac{F(f, h)}{F(f, g)} f . m, \quad m_{\cdot_{F}} f=\frac{F(h, f)}{F(g, f)} m . f
$$

for all $f, g, h \in G$ and $m \in{ }^{g} M^{h}$. It is easy to verify that $M_{F}$ is indeed a $\left(k G, \Phi_{F}\right)$-Majid bimodule. We say that the $(k G, \Phi)$-Majid bimodule $M$ and the $\left(k G, \Phi^{\prime}\right)$-Majid bimodule $N$ are gauge equivalent, if there exists a gauge transformation $F$ on $G$ such that $\Phi^{\prime}=\Phi_{F}$ and $M_{F} \cong N$ as $\left(k G, \Phi^{\prime}\right)$-Majid bimodules. If $F$ is a gauge transformation on $G$, then so is $F^{-1}$ and we have

$$
\left(M_{F}\right)_{F^{-1}}=M=\left(M_{F^{-1}}\right)_{F} .
$$

If $F^{\prime}$ is another gauge transformation on $G$, then so is the product $F F^{\prime}$ and we have

$$
\left(M_{F}\right)_{F^{\prime}}=M_{F F^{\prime}}
$$

By (4.6)-(4.7), it is clear that the gauge equivalence is an equivalence relation in the family of Majid bimodules over the group $G$ with arbitrary 3-cocycles.

According to Theorem 3.3, the gauge equivalence of Majid bimodules should be described by a suitable transformation of the family of admissible collections of projective representations. Before giving this transformation, we need to fix some notations. For each conjugacy class $C$ of $G$, fix an element $g(C) \in C$. Then we adopt all the notations as given before Theorem 3.3. Let $\left(M_{C}\right)_{C \in \mathcal{C}}$ be a $\Phi$-collection. Suppose that $F$ is a gauge transformation on $G$. Let $\Phi_{F}$ be as (4.4). We build a $\Phi_{F}$-collection $\left(M_{C}^{F}\right)_{C \in \mathcal{C}}$ by these data. The space of $M_{C}^{F}$ is the same as $M_{C}$ and the action is given by

$$
f \triangleright_{F} m=\frac{F\left(f, f^{-1}\right)}{F(f, g(C)) F\left(f g(C), f^{-1}\right)} f \triangleright m
$$

for all $f \in Z_{C}$ and $m \in M_{C}$. Let $\Phi^{\prime}$ be another 3-cocycle on $G$ and $\left(N_{C}\right)_{C \in \mathcal{C}}$ a $\Phi^{\prime}$-collection. The two admissible collections $\left(M_{C}\right)_{C \in \mathcal{C}}$ and $\left(N_{C}\right)_{C \in \mathcal{C}}$ are said to be gauge equivalent, if there exists a gauge transformation $F$ on $G$ such that $\Phi^{\prime}=\Phi_{F}$ and $M_{C}^{F} \cong N_{C}$ as $\tilde{\Phi}^{\prime}{ }^{-}$-representations of $Z_{C}$ for all $C$. It is easy to verify that this gauge equivalence has properties like (4.6)-(4.7) and therefore it is also an equivalence relation.

The equation (4.8) suggests that the gauge equivalence of admissible collections can be described by the projective equivalence of projective representations of groups. Now we need to recall some more definitions and facts. 
Two projective representations $\rho_{i}: G \longrightarrow G L\left(V_{i}\right), \quad i=1,2$, are said to be projectively equivalent if there exists a mapping $\mu: G \longrightarrow k^{*}$ with $\mu(\epsilon)=1$ and a vector space isomorphism $\phi: V_{1} \longrightarrow V_{2}$ such that

$$
\rho_{2}(g)=\mu(g) \phi \rho_{1}(g) \phi^{-1}
$$

for all $g \in G$. If $\mu(g)=1$ for all $g \in G$, then $\rho_{1}$ and $\rho_{2}$ are said to be linearly equivalent. We declare that the isomorphisms we have used so far are linear equivalence by default. Let $\rho_{i}, \quad i=1,2$, be an $\alpha_{i}$-representation. Then $\rho_{1}$ is projectively (respectively, linearly) equivalent to $\rho_{2}$ implies that $\alpha_{1}$ is cohomologous (respectively, equal) to $\alpha_{2}$. On the other hand, if $\rho_{1}$ is an $\alpha_{1}$ representation on the space $V$, then for any 2-cocycle $\alpha_{2}$ that is cohomologous to $\alpha_{1}$ there exists an $\alpha_{2}$-representation $\rho_{2}$ on $V$ which is projectively equivalent to $\rho_{1}$. In particular, if $\alpha_{1}$ is a 2-coboundary, then $\rho_{1}$ is projectively equivalent to a linear representation.

Let $F$ be a gauge transformation on $G,\left(M_{C}\right)_{C \in \mathcal{C}}$ a $\Phi$-collection, and $\left(M_{C}^{F}\right)_{C \in \mathcal{C}}$ the corresponding $\Phi^{\prime}$-collection under gauge transformation. For each $C \in \mathcal{C}$, set a mapping $\mu_{C}^{F}: Z_{C} \longrightarrow k^{*}$ by

$$
\mu_{C}^{F}(f)=\frac{F\left(f, f^{-1}\right)}{F(f, g(C)) F\left(f g(C), f^{-1}\right)}
$$

for all $f \in Z_{C}$. Clearly, for all $C \in \mathcal{C}, M_{C}$ and $M_{C}^{F}$ are projectively equivalent as projective representations of $Z_{C}$. In particular, the mappings $\mu_{C}^{F}$ are determined by $F$ in a uniform way. We say the two collections $\left(M_{C}\right)_{C \in \mathcal{C}}$ and $\left(M_{C}^{F}\right)_{C \in \mathcal{C}}$ of projective representations are $F$-uniformly projective equivalent. In general, when the gauge transformation is not explicit, we simply say two collections are uniformly projective equivalent. In this terminology, a $\Phi$-collection $\left(M_{C}\right)_{C \in \mathcal{C}}$ is gauge equivalent to a $\Phi^{\prime}$-collection $\left(N_{C}\right)_{C \in \mathcal{C}}$ if and only if there exists a gauge transformation $F$ on $G$ such that $\Phi^{\prime}=\Phi \partial F$ and that the two collections $\left(M_{C}\right)_{C \in \mathcal{C}}$ and $\left(N_{C}\right)_{C \in \mathcal{C}}$ are $F$-uniformly projective equivalent.

Now we are ready to describe the gauge equivalence of graded Majid algebras. For a Majid bimodule $M$ over $G$, let $\left(M_{C}\right)_{C \in \mathcal{C}}$ be the corresponding admissible collection. We have shown in [13] that one can associate to $M$ a unique Hopf quiver and construct a unique graded Majid algebra, denoted by $H(M)$, on the path coalgebra as in Proposition 4.1. Similar to Nichols' bialgebras of type one [20], we define $B(M)$ to be the sub Majid algebra of $H(M)$ generated in degrees 0 and 1 , that is the smallest sub Majid algebra of $H(M)$ containing $G$ and $M$.

Theorem 4.3. Let $M, N$ be Majid bimodules over $G$. Then the following are equivalent: 
(1) $H(M)$ and $H(N)$ are gauge equivalent.

(2) $B(M)$ and $B(N)$ are gauge equivalent.

(3) $M$ and $N$ are gauge equivalent.

(4) $\left(M_{C}\right)_{C \in \mathcal{C}}$ and $\left(N_{C}\right)_{C \in \mathcal{C}}$ are gauge equivalent.

(5) $\left(M_{C}\right)_{C \in \mathcal{C}}$ and $\left(N_{C}\right)_{C \in \mathcal{C}}$ are uniformly projective equivalent.

Proof. (3) $\Leftrightarrow(4)$ and (4) $\Leftrightarrow(5)$ are direct from definitions. (1) $\Leftrightarrow(3)$ and $(2) \Leftrightarrow(3)$ are similar, so we prove only $(1) \Leftrightarrow(3)$.

First suppose that $M$ is a $(k G, \Phi)$-Majid bimodule and $N$ is a $\left(k G, \Phi^{\prime}\right)$ Majid bimodule and that they are gauge equivalent. Then there exists a gauge transformation $F$ on $G$ such that $\Phi^{\prime}=\Phi_{F}$ and $M_{F} \cong N$ as $\left(k G, \Phi^{\prime}\right)$ Majid bimodules. Clearly $F$ can be lifted as a gauge transformation on $H(M)$ which concentrates at degree zero. By the defintion of $H(M)_{F}$, one sees that it is exactly the graded Majid algebra corresponding to the Majid bimodule $M_{F}$. Now we have $H(M)_{F}=H\left(M_{F}\right) \cong H(N)$ by Proposition 4.1.

Conversely, suppose that $H(M)$ and $H(N)$ are gauge equivalent. Then there exists a gauge transformation $F$ on $H(M)$ such that $H(M)_{F}$ is isomorphic to $H(N)$. Since $H(M)_{F}$ is graded, then by (4.1) we have that $F$ concentrates at degree zero part. When we restrict $F$ to degree zero part, the equation (4.2) becomes (4.4), so it is actually a gauge transformation on $G$. The isomorphism map preserves the coradical filtration. In particular its restriction to the coradicals, i.e. the degree zero parts, is an isomorphism. Without loss of generality, we may assume the map preserves gradation and the restriction on degree zero is identity. Assume that $M$ is a $(k G, \Phi)$-Majid bimodule and $N$ and a $\left(k G, \Phi^{\prime}\right)$-Majid bimodule. Now by (4.2) we have $\Phi^{\prime}=\Phi_{F}$. The isomorphism $H(M)_{F} \cong H(N)$ also induces the isomorphism of degree one parts as $\left(k G, \Phi^{\prime}\right)$-Majid bimodules. Since $H(M)_{F}=H\left(M_{F}\right)$, we have $M_{F} \cong N$.

The previous result can be generalized to the gauge equivalence for more general graded pointed Majid algebras. For example, The equivalent conditions (3)-(5) are always necessary for two graded pointed Majid algebras to be gauge equivalent. Now it is interesting to remark that, the isomorphism is to the gauge equivalence for graded pointed Majid algebras is almost as much as the linear equivalence is to the projective equivalence for projective representations of groups.

We conclude this section with a corollary of the previous theorem, which deals with the special case when a graded pointed Majid algebra is gauge equivalent to a usual Hopf algebra.

Corollary 4.4. Suppose that $M$ is a $(k G, \Phi)$-Majid bimodule. Let $\left(M_{C}\right)_{C \in \mathcal{C}}$, $H(M)$ and $B(M)$ be as before. Then the following are equivalent: 
(1) $H(M)$ is gauge equivalent to a Hopf algebra.

(2) $B(M)$ is gauge equivalent to a Hopf algebra.

(3) $M$ is gauge equivalent to a $k G$-Hopf bimodule.

(4) $\Phi$ is a 3-coboundary.

(5) $\left(M_{C}\right)_{C \in \mathcal{C}}$ is gauge equivalent to an admissible collection of linear representations.

(6) $\left(M_{C}\right)_{C \in \mathcal{C}}$ is uniformly projective equivalent to a collection of linear representations.

Proof. We prove only $(1) \Leftrightarrow(4)$, since the other equivalences are direct consequence of Theorem 4.3. Suppose that $\Phi$ is a 3 -coboundary, then there exists a 2-cochain $F$ such that $\Phi=\partial F$. Note that $F$ can be chosen in the gauge transformations on $G$. By (4.4) we have that $\Phi_{F^{-1}}$ is the trivial 3cocycle and so the Majid bimodule $M_{F^{-1}}$ defined by by (4.5) is a $k G$-Hopf bimodule. Now it is clear that $H(M)_{F^{-1}}=H\left(M_{F^{-1}}\right)$ is a Hopf algebra. That is, $H(M)$ is gauge equivalent to a Hopf algebra. For the converse, suppose that $H(M)$ is gauge equivalent to a Hopf algebra $H$. There exists a gauge transformation $F$ on $H(M)$ such that $H(M)_{F} \cong H$. Note that the reassociator on $H$ is trivial, and then by (4.2) we have that the 3 -cocycle $\Phi_{F}$ on $G$ is trivial, which means that $\Phi=\partial F^{-1}$. We are done.

\section{MAJid Algebras OVER $\mathbb{Z}_{2}$}

In this section, we apply the constructed machinery to give some examples and a classification of finite-dimensional graded pointed Majid algebras over the simplest nontrivial group $\mathbb{Z}_{2}$.

For the construction of graded Majid algebras on path coalgebras, we have to calculate the product of paths. This can be done by the quantum shuffle product as shown in [13]. So firstly we need to recall the the product formula in [5].

Suppose that $Q$ is a Hopf quiver with a necessary $k Q_{0}$-Majid bimodule structure on $k Q_{1}$. By $Q_{l}$ we denote the set of paths of length $l$. Let $p \in Q_{l}$ be a path. An $n$-thin split of it is a sequence $\left(p_{1}, \cdots, p_{n}\right)$ of vertices and arrows such that the concatenation $p_{n} \cdots p_{1}$ is exactly $p$. These $n$-thin splits are in one-to-one correspondence with the $n$-sequences of $(n-l) 0$ 's and $l$ 1's. Denote the set of such sequences by $D_{l}^{n}$. Clearly $\left|D_{l}^{n}\right|=\left(\begin{array}{l}n \\ l\end{array}\right)$. For $d=\left(d_{1}, \cdots, d_{n}\right) \in D_{l}^{n}$, the corresponding $n$-thin split is written as $d p=\left((d p)_{1}, \cdots,(d p)_{n}\right)$, in which $(d p)_{i}$ is a vertex if $d_{i}=0$ and an arrow if $d_{i}=1$. Let $\alpha=a_{m} \cdots a_{1}$ and $\beta=b_{n} \cdots b_{1}$ be paths of length $m$ and $n$ respectively. Let $d \in D_{m}^{m+n}$ and $\bar{d} \in D_{n}^{m+n}$ the complement sequence which is obtained from $d$ by replacing each 0 by 1 and each 1 by 0 . Define an 
element

$$
(\alpha \cdot \beta)_{d}=\left[(d \alpha)_{m+n} \cdot(\bar{d} \beta)_{m+n}\right] \cdots\left[(d \alpha)_{1} \cdot(\bar{d} \beta)_{1}\right]
$$

in $k Q_{m+n}$, where $\left[(d \alpha)_{i} \cdot(\bar{d} \beta)_{i}\right]$ is understood as the action of $k Q_{0}$-Majid bimodule on $k Q_{1}$ and these terms in different brackets are put together by cotensor product, or equivalently concatenation. In terms of these notations, the formula of the product of $\alpha$ and $\beta$ is given as follows:

$$
\alpha \cdot \beta=\sum_{d \in D_{m}^{m+n}}(\alpha \cdot \beta)_{d} .
$$

We should remark that, for general Majid algebras, the product is not associative. So the order must be concerned for the product of more than two terms.

Convention 5.1. For an arbitrary path $p$ and an integer $n \geq 3$, let $p^{\vec{n}}$

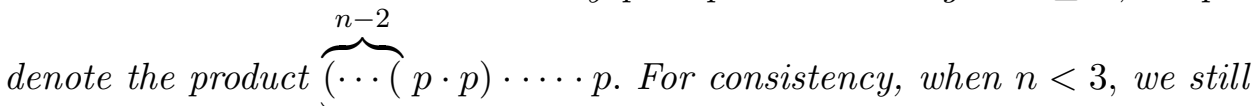
use the notation $p^{\vec{n}}$ although there is no risk of associative problem.

For simplicity, we assume that $k$ is the field of complex numbers in the rest of the paper. Let $g$ denote the generator of the group $\mathbb{Z}_{2}$. There is only one nontrivial 3-cocycle $\Phi$ on $\mathbb{Z}_{2}$ by (2.5)-(2.6). That is, the value of $\Phi$ on the triple $(g, g, g)$ is -1 and on any other triples is 1 . The induced 2-cocycle $\tilde{\Phi}_{\epsilon}$ is trivial, while $\tilde{\Phi}_{g}$ is nontrivial satisfying $\tilde{\Phi}_{g}(g, g)=-1$. For the classification of $\left(k \mathbb{Z}_{2}, \Phi\right)$-Majid bimodules, it suffices to study the representation theory of $k^{\tilde{\Phi}_{\epsilon}} \mathbb{Z}_{2}=k \mathbb{Z}_{2}$ and $k^{\tilde{\Phi}_{g}} \mathbb{Z}_{2}$. The former is well-known. For the latter, let $\star$ denote the product and then we have

$$
g \star g=\tilde{\Phi}_{g}(g, g) g^{2}=-\epsilon .
$$

Therefore the action of $g$ on a linear space is always diagonalizable and so simple $k^{\tilde{\Phi}_{g}} \mathbb{Z}_{2}$-modules are 1-dimensional. Assume $V=k v$ is a simple $k^{\tilde{\Phi}_{g}} \mathbb{Z}_{2}$-module and $g \triangleright v=\lambda v$. It follows from (5.2) that $\lambda^{2}=-1$, hence $\lambda= \pm i$. There are two simple $k^{\tilde{\Phi}_{g}} \mathbb{Z}_{2}$-modules, denoted by $S(+i)$ and $S(-i)$ respectively. An arbitrary $k^{\tilde{\Phi}_{g}} \mathbb{Z}_{2}$-module is of the form $m S(+i) \oplus n S(-i)$ where $m, n$ are non-negative integers.

Let $R_{1}=g$ be a ramification datum of $\mathbb{Z}_{2}$. Then the associated Hopf quiver $Q\left(\mathbb{Z}_{2}, R_{1}\right)$, denoted by $\Gamma^{1}$ for short, is as follows:

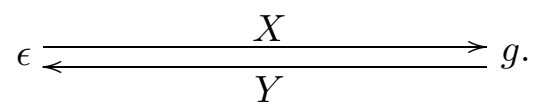

Conversely, this is the unique way for $\Gamma^{1}$ to be viewed as a Hopf quiver. So the set of graded Majid algebra structures on the path coalgebra $k \Gamma^{1}$ is determined by all the possible Majid bimodule structures on $M=k X \oplus k Y$ over the group $\mathbb{Z}_{2}$. We are interested in nontrivial Majid algebras (i.e., not 
gauge equivalent to Hopf algebras), so we take the nontrivial 3-cocycle $\Phi$ on $\mathbb{Z}_{2}$ as above. Note that $\Phi$ is not a 3 -coboundary by the well-known fact $H^{3}\left(\mathbb{Z}_{2}, k^{*}\right)=\mathbb{Z}_{2}$. By Theorem 3.3, it is enough to consider the $k^{\tilde{\Phi}_{g}} Z_{2}$-module structure on ${ }^{g} M^{\epsilon}=k X$, which is either $S(+i)$ or $S(-i)$ by the previous argument. It is clear that these two modules are not gauge equivalent, since by (4.8) they are stable under any gauge transformation of $\mathbb{Z}_{2}$. Hence there are two non-trivial graded Majid algebras on $k \Gamma^{1}$ up to gauge equivalence described as follows.

Example 5.2. The one induced by $S(+i)$, denoted by $k \Gamma^{1}(+i)$. By Theorem 3.3, we may extend $S(+i)$ to a Majid bimodule on $M$ as:

$$
X . g=Y, \quad Y . g=-X, \quad g . X=i Y, \quad g . Y=i X .
$$

We have the following interesting product formulae of paths:

(5.4) $X \cdot X=(1+i) Y X, \quad Y X \cdot X=-i X Y X, \quad Y X \cdot Y X=X Y X \cdot X=0$.

Consider the sub Majid algebra generated by the vertices and arrows of $\Gamma^{1}$. By (5.3) it is actually generated by $g$ and $X$. Since $((X \cdot X) \cdot X) \cdot X)=0$ and the products in other orders differ only by nonzero scalars according to the definition of Majid algebras, then along with (5.4) it is easy to show that all the paths of length less than 4 constitute a basis for this sub Majid algebra. In particular it is 8-dimensional. Let $M_{+}(8)$ denote this Majid algebra.

Example 5.3. The one induced by $S(-i)$, denoted by $k \Gamma^{1}(-i)$. We may extend $S(-i)$ to a Majid bimodule on $M$ as:

$$
X . g=Y, \quad Y . g=-X, \quad g . X=-i Y, \quad g . Y=-i X .
$$

We have the following similar product formulae of paths:

$$
X \cdot X=(1-i) Y X, \quad Y X \cdot X=i X Y X, \quad Y X \cdot Y X=X Y X \cdot X=0 .
$$

Similarly the sub Majid algebra generated by the vertices and arrows of $\Gamma^{1}$ is 8-dimensional and all the paths of length less than 4 constitute a basis. Let $M_{-}(8)$ denote this Majid algebra.

Now let $R_{2}=2 g$ be a ramification datum of $\mathbb{Z}_{2}$ and $\Gamma^{2}$ the associated Hopf quiver:

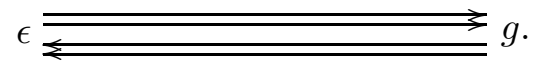

Let $X_{1}, X_{2}$ denote the two arrows going from $\epsilon$ to $g$, and $Y_{1}, Y_{2}$ those in the converse direction. The set of nontrivial graded Majid algebra structures on $k \Gamma^{2}$ is determined by all the $k^{\tilde{\Phi}_{g}} Z_{2}$-module structures on ${ }^{g} M^{\epsilon}=$ $k X_{1} \oplus k X_{2}$, which are $S(+i) \oplus S(+i), S(-i) \oplus S(-i)$, and $S(+i) \oplus S(-i)$. Hence there are three non-trivial graded Majid algebras on $k \Gamma^{2}$, denoted by $k \Gamma^{2}(++), k \Gamma^{2}(--)$, and $k \Gamma^{2}( \pm)$ respectively. 
Example 5.4. The product of the Majid algebra $k \Gamma^{2}( \pm)$ is determined by the Majid bimodule extended by $S(+i) \oplus S(-i)$. Without loss of generality, we may assume

$$
\begin{aligned}
& X_{1} . g=Y_{1}, \quad Y_{1} \cdot g=-X_{1}, \quad g \cdot X_{1}=i Y_{1}, \quad g \cdot Y_{1}=i X_{1}, \\
& X_{2} \cdot g=Y_{2}, \quad Y_{2} \cdot g=-X_{2}, \quad g \cdot X_{2}=-i Y_{2}, \quad g \cdot Y_{2}=-i X_{2} .
\end{aligned}
$$

Then we have product formulae like (5.4) by replacing $X, Y$ as $X_{1}, Y_{1}$ and (5.6) by replacing $X, Y$ as $X_{2}, Y_{2}$. We also have

$$
X_{1} \cdot X_{2}=Y_{1} X_{2}-i Y_{2} X_{1}, \quad X_{2} \cdot X_{1}=i Y_{1} X_{2}+Y_{2} X_{1} .
$$

This implies that

$$
X_{1} \cdot X_{2}=-i X_{2} \cdot X_{1}
$$

Consider the sub Majid algebra generated by the vertices and arrows of $\Gamma^{2}$ and denote it by $M(32)$. By (5.7)-(5.8) it is actually generated by $g, X_{1}, X_{2}$. According to the previous product formulae, it is not difficult to verify that

$$
\left\{g^{l} \cdot\left(X_{1}^{\vec{m}} \cdot X_{2}^{\vec{n}}\right) \mid 0 \leq m, n \leq 3, l=0,1\right\}
$$

constitutes a basis for $M(32)$. In particular $M(32)$ is 32-dimensional.

We derive some product formulae for $k \Gamma^{2}(++)$ and $k \Gamma^{2}(--)$ in the same manner.

Example 5.5. Extend $S(+i) \oplus S(+i)$ to the Majid bimodule as follows:

$$
\begin{aligned}
& X_{1} . g=Y_{1}, \quad Y_{1} . g=-X_{1}, \quad g . X_{1}=i Y_{1}, \quad g . Y_{1}=i X_{1}, \\
& X_{2} \cdot g=Y_{2}, \quad Y_{2} \cdot g=-X_{2}, \quad g \cdot X_{2}=i Y_{2}, \quad g \cdot Y_{2}=i X_{2} \text {. }
\end{aligned}
$$

Then we have product formulae for $k \Gamma^{2}(++)$ like (5.4) by replacing $X, Y$ as $X_{j}, Y_{j}, j=1,2$. We also have

$$
X_{1} \cdot X_{2}=Y_{1} X_{2}+i Y_{2} X_{1}, \quad X_{2} \cdot X_{1}=i Y_{1} X_{2}+Y_{2} X_{1} .
$$

This implies that

$$
X_{2} \cdot X_{1}-i X_{1} \cdot X_{2}=2 Y_{2} X_{1}
$$

Example 5.6. Extend $S(-i) \oplus S(-i)$ to the Majid bimodule as follows:

$$
\begin{array}{llll}
X_{1} . g=Y_{1}, & Y_{1} \cdot g=-X_{1}, & g \cdot X_{1}=-i Y_{1}, & g \cdot Y_{1}=-i X_{1}, \\
X_{2} . g=Y_{2}, & Y_{2} \cdot g=-X_{2}, & g \cdot X_{2}=-i Y_{2}, & g \cdot Y_{2}=-i X_{2} .
\end{array}
$$

Then we have product formulae for $k \Gamma^{2}(--)$ like (5.6) by replacing $X, Y$ as $X_{j}, Y_{j}, j=1,2$. We also have

$$
X_{1} \cdot X_{2}=Y_{1} X_{2}-i Y_{2} X_{1}, \quad X_{2} \cdot X_{1}=Y_{2} X_{1}-i Y_{1} X_{2} .
$$


This implies that

$$
X_{1} \cdot X_{2}+i X_{2} \cdot X_{1}=2 Y_{1} X_{2}
$$

We are interested in their sub Majid algebras generated by the vertices and arrows of $\Gamma^{2}$. It turns out that the situation is quite different from that of $k \Gamma^{2}( \pm)$.

Proposition 5.7. The sub Majid algebras of $k \Gamma^{2}(++)$ and $k \Gamma^{2}(--)$ generated by the set of vertices and arrows of $\Gamma^{2}$ are infinite-dimensional.

Proof. We only prove the claim for $k \Gamma^{2}(++)$. The other is similar. By (5.14), the path $Y_{2} X_{1}$ lies in the sub Majid algebra. Calculate the power $\left(Y_{2} X_{1}\right)^{\vec{n}}$. By (5.1) and induction on $n$, it is not hard to show that

$$
\left(Y_{2} X_{1}\right)^{\vec{n}}=n ! \overbrace{Y_{2} X_{1} \cdots Y_{2} X_{1}}^{\mathrm{n} \text { copies of } Y_{2} X_{1}}+\text { other paths. }
$$

This implies that $\left(Y_{2} X_{1}\right)^{\vec{n}} \neq 0$ for all $n$ and that the set $\left\{\left(Y_{2} X_{1}\right)^{\vec{n}} \mid n \geq 0\right\}$ is linearly independent. We are done.

An arbitrary ramification datum of $\mathbb{Z}_{2}$ is of the form $R=m \epsilon+n g$. The associated Hopf quiver looks like the following

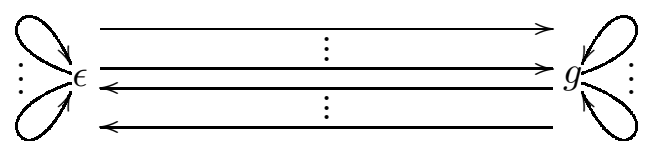

where there are $m$ loops attached to both vertices, and there are $n$ arrows going from $\epsilon$ to $g$ and vice versa. As before, one can apply the representation theory of twisted group algebras to get Majid bimodule structures on the space of arrows and then the product formulae for the graded Majid algebras.

Now we are ready to give our first classification result for finite-dimensional graded pointed Majid algebras with a help of the quiver framework.

Theorem 5.8. Assume that $H$ is a finite-dimensional graded pointed nontrivial Majid algebra whose set of group-likes is equal to $\mathbb{Z}_{2}$. Then $H$ is isomorphic to one of $\left\{M_{+}(8), M_{-}(8), M(32)\right\}$. Moreover, $M_{+}(8)$ is not gauge equivalent to $M_{-}(8)$.

Proof. By assumption we can write $H=\oplus_{n \geq 0} H_{n}$ where $H_{0}=k \mathbb{Z}_{2}$. Let $\Phi$ denote the reassociator of $H$. Since $\Phi$ concentrates at $M_{0}$ by the graded condition, it is a 3 -cocycle on $\mathbb{Z}_{2}$. Moreover $\Phi$ is nontrivial by Corollary 4.4, since the Majid algebra $H$ is not gauge equivalent to a Hopf algebra.

By the Gabriel type theorem for pointed Majid algebras, there exists a unique Hopf quiver of the form $Q\left(\mathbb{Z}_{2}, R\right)$, where $R$ is a ramification datum of $\mathbb{Z}_{2}$, such that $H$ is isomorphic to a large sub Majid algebra of a graded 
Majid algebra structure on $k Q\left(\mathbb{Z}_{2}, R\right)$. Recall that "large" means containing the subspace $k Q\left(\mathbb{Z}_{2}, R\right)_{0} \oplus k Q\left(\mathbb{Z}_{2}, R\right)_{1}$. In the following we always view $H$ as a sub Majid algebra of $k Q\left(\mathbb{Z}_{2}, R\right)$.

We claim that the Hopf quiver $Q\left(\mathbb{Z}_{2}, R\right)$ of $H$ can only be $\Gamma^{1}$ or $\Gamma^{2}$. Assume that $R=m \epsilon+n g$. If $m>0$, then there is at least one loop attached to $\epsilon$. Let $\ell$ denote the loop and we have $\ell \in H$ since $H$ is large. Now we calculate $\ell^{\vec{s}}$ by making use of (5.1). By induction on $s$, we get $\ell^{\vec{s}}=s ! \overbrace{\ell \cdots \ell}^{\text {n }}$. It follows that the infinite set $\left\{\ell^{s} \mid s \geq 0\right\}$ is linearly independent, which contradicts the finiteness assumption of $H$. If $n>2$, then there are at least three arrows going from $\epsilon$ to $g$. That means $k Q\left(\mathbb{Z}_{2}, R\right)$ must contain at least a sub Majid algebra as $k \Gamma^{2}(++)$ or $k \Gamma^{2}(--)$. By Proposition 5.7, the minimal large sub Majid algebra (generated by the set of vertices and arrows) is already infinite-dimensional, which contradicts the finiteness assumption again. Therefore, $m=0$ and $n \leq 2$, which justifies the claim.

Now by Examples (5.2)-(5.4) and Proposition (5.7) we can conclude that $H$ must be a large sub Majid algebra of $k \Gamma^{1}(+i), k \Gamma^{1}(-i)$ or $k \Gamma^{2}( \pm)$. Therefore $H$ must contain one of $\left\{M_{+}(8), M_{-}(8), M(32)\right\}$ as a sub Majid algebra, since these are minimal large sub Majid algebras. It remains to prove that $M_{+}(8), M_{-}(8)$, and $M(32)$ are the only finite-dimensional large sub Majid algebras of $k \Gamma^{1}(+i), k \Gamma^{1}(-i)$, and $k \Gamma^{2}( \pm)$ respectively. We will verify this case by case.

Assume that $H \supsetneq M_{+}(8)$ is a graded sub Majid algebra of $k \Gamma^{1}(+i)$. Then $H$ must contain paths of length $\geq 4$. Consider the coproduct of such paths, then one can see that there exist paths of length exactly equal to 4. So we have $Y X Y X \in H$. Calculate $(Y X Y X)^{\vec{s}}$. By induction on $s$ we have $(Y X Y X)^{\vec{s}}=\overbrace{Y X Y X \cdots Y X Y X}^{s \text { terms of } Y X Y X}$. It follows that $H$ must be infinitedimensional. Similarly we can prove the claim for $M_{-}(8)$.

Assume that $H \supsetneq M(32)$ is a finite-dimensional graded sub Majid algegra of $k \Gamma^{2}( \pm)$. That means $g, X_{1}, X_{2}$ can not fully generate $H$. As the above argument, paths with segments like $Y_{1} X_{1} Y_{1} X_{1}$ or $Y_{2} X_{2} Y_{2} X_{2}$ can not occur in $H$. Consider the linear combinations of $Y_{2} X_{1}$ and $Y_{1} X_{2}$ occurs in $H$. If all of them are linearly dependent to $Y_{1} X_{2}-i Y_{2} X_{1}=X_{1} \cdot X_{2}$, then $g, X_{1}, X_{2}$ generate $H$. There must be linear combinations of $Y_{2} X_{1}$ and $Y_{1} X_{2}$ which are linearly independent to $Y_{1} X_{2}-i Y_{2} X_{1}$. In that case, we have $Y_{2} X_{1} \in H$. Now calculate $\left(Y_{2} X_{1}\right)^{\vec{s}}$ and get a formula like (5.19), then we arrive at a contradiction.

The second claim is a direct consequence of Theorem 4.3 and the fact that $S(+i)$ is not gauge equivalent to $S(-i)$. The proof is completed. 
We conclude the paper with some remarks. Firstly, if we take $\Phi$ to be the trivial 3 -cocycle on $\mathbb{Z}_{2}$ and carry out the previous procedure, then we get graded pointed Hopf algebras. To get finite-dimensional graded pointed Hopf algebras over $Z_{2}$, we need to work on Hopf quivers of the form $Q\left(\mathbb{Z}_{2}, n g\right)$. For all integers $n$, one can recover the well-known Nichols' Hopf algebras [20] of dimension $2^{n+1}$ on the Hopf quivers $Q\left(\mathbb{Z}_{2}, n g\right)$, which provide a classification of finite-dimensional graded pointed Hopf algebras over $\mathbb{Z}_{2}$. For those who are familiar with this result, Theorem 5.8 seems weird at first sight. We believe that our approach gives an acceptable representationtheoretic explanation for this phenomenon.

Secondly, note that the notions of Majid algebras and quasi-Hopf algebras are dual to each other. In particular, the dual of a finite-dimensional pointed Majid algebra is an elementary quasi-Hopf algebras and vice versa. Our method can be dualized to get the corresponding results on quasi-Hopf algebras by making use of path algebras and quasi-Hopf bimodules (see [24]) over the dual of group algebras. For example, the dual of Theorem 5.8 recovers Etingof and Gelaki's classification of elementary graded non-trivial quasi-Hopf algebras with radical of codimension 2 in [8]. It is easy to check that the dual of our Majid algebras $M_{+}(8), M_{-}(8), M(32)$ are isomorphic to their quasi-Hopf algebras $H_{+}(8), H_{-}(8), H(32)$ respectively. Moreover, the coproducts of $H_{+}(8), H_{-}(8), H(32)$ can be given by the quasi-bicomodules of the quasi-Hopf bimodules (i.e., the dual of our Majid bimodules in Examples 5.2-5.4) in a conceptual way. It should be pointed out that these two approaches are not just simply dual to each other. Our approach emphasizes the coalgebraic side and the Majid algebras are constructed as sub algebras, while the approach of Etingof and Gelaki emphasizes the algebraic side and their quasi-Hopf algebras are constructed as quotient algebras. It is better to combine both, especially when we want to determine a finitedimensional quasi-quantum group. For example, in the proof of Theorem 5.8 , our approach gives easily the lower bound of the dimension of a pointed Majid algebra in concern, while Etingof and Gelaki's approach gives easily the upper bound of the dimension of the quasi-Hopf algebra (=the dual of our Majid algebra), then the claim follows from the dimension comparison.

Finally, we would like to remark that the quiver presentation for Majid algebras and quasi-Hopf algebras is very useful in studying their structure and (co)representation theory. For example, the quiver presentations of $M_{+}(8)$ and $M_{-}(8)$ indicate that they are monomial in the sense of [3]. Then similar to [loc. cit.], one can show by quiver representation theory that their comodule categories have a very interesting property, namely there are only finitely many indecomposable objects up to isomorphism. This phenomenon 
is called finite representation type and plays very import role in the representation theory of algebras (see [1]). The study of such finite-dimensional algebras has been a central theme all along. Thus in our setting, a very natural problem is to give a complete classification of finite-dimensional pointed Majid algebras of finite corepresentation type in the sense of [18]. According to the Tannakian formalism, it amounts to a classification of the class of finite tensor categories in which there are finitely many indecomposable objects up to isomorphism with mild conditions. We believe that this should be of interest for the theory of finite tensor categories as well. This problem is dealt with in another paper [14].

Acknowledgement: The research was supported by the National NSF of China under grant number 10601052. The author thanks Gongxiang Liu and $\mathrm{Yu}$ Ye for useful discussions.

\section{REFERENCES}

[1] I. Assem, D. Simson, A. Skowronski, Elements of the Representation Theory of Associative Algebras. Vol. 1. Techniques of Representation Theory. London Mathematical Society Student Texts, 65. Cambridge University Press, Cambridge, 2006.

[2] D. Calaque, P. Etingof, Lectures on tensor categories, math.QA/0401246.

[3] X.-W. Chen, H.-L. Huang, Y. Ye, P. Zhang, Monomial Hopf algebras, J. Algebra 275 (2004) 212-232.

[4] C. Cibils, M. Rosso, Algèbres des chemins quantiques, Adv. Math. 125 (1997) 171199.

[5] C. Cibils, M. Rosso, Hopf quivers, J. Algebra 254 (2002) 241-251.

[6] R. Dijkgraaf, V. Pasquier, P. Roche, Quasi Hopf algebras, group cohomology and orbifold models, in: Recent Advances in Field Theory, Annecy-le-Vieux, 1990, Nucl. Phys. B Proc. Suppl. 18B (1991) 60-72.

[7] V.G. Drinfeld, Quasi-Hopf algebras, Leningrad Math. J. 1 (1990) 1419-1457.

[8] P. Etingof, S. Gelaki, Finite dimensional quasi-Hopf algebras with radical of codimension 2, Math. Res. Lett. 11 (2004) 685-696.

[9] P. Etingof, S. Gelaki, On radically graded finite-dimensional quasi-Hopf algebras, Mosc. Math. J. 5(2) (2005) 371-378.

[10] P. Etingof, S. Gelaki, Liftings of graded quasi-Hopf algebras with radical of prime codimension, J. Pure Appl. Algebra 205(2) (2006) 310-322.

[11] S. Gelaki, Basic quasi-Hopf algebras of dimension $n^{3}$, J. Pure Appl. Algebra 198(1-3) (2005) 165-174.

[12] F. Hausser, F. Nill, Integral theory for quasi-Hopf algebras, math.QA/9904164.

[13] H.-L. Huang, Quiver approaches to quasi-Hopf algebras, J. Math. Phys., to appear, arXiv:0902.1620.

[14] H.-L. Huang, G. Liu, Y. Ye, Quviers, quasi-quantum groups and finite tensor categories, in preparation.

[15] G. Karpilovsky, Projecive Representations of Finite Groups, Marcel Dekker, New York, 1985. 
[16] C. Kassel, Quantum Groups, Graduate Texts in Math. 155, Springer-Verlag, New York, 1995.

[17] R.G. Larson, M.E. Sweedler, An associative orthogonal bilinear form for Hopf algebras, Amer. J. Math. 91 (1969) 149-166.

[18] G. Liu, F. Li, Pointed Hopf algebras of finite corepresentation type and their classifications, Proceedings of AMS, 135(3) (2007) 649-657.

[19] S. Majid, Foundations of Quantum Group Theory, Cambridge University Press, Cambridge, 1995.

[20] W.D. Nichols, Bialgebras of type one, Communications in Algebra 6(15) (1978) 15211552.

[21] M. Rosso, Groupes quantiques et algèbres de battage quantiques, C. R. Acad. Sci. Paris 320 (1995) 145-148.

[22] M. Rosso, Quantum groups and quantum shuffles, Invent. Math. 133 (1998) 399-416.

[23] P. Schauenburg, Hopf modules and Yetter-Drinfeld modules, J. Algebra 169 (1994) 874-890.

[24] P. Schauenburg, Hopf modules and the double of a quasi-Hopf algebra, Trans. Amer. Math. Soc. 354(8) (2002) 3349-3378.

[25] S. Shnider, S. Sternberg, Quantum Groups: From Coalgebras to Drinfeld Algebras, International Press Inc., Boston, 1993.

[26] D. Yetter, Quantum groups and representations of monoidal categories, Math. Proc. Camb. Phil. Soc. 108 (1990) 261-290.

School of Mathematics, Shandong University, Jinan 250100, P. R. China

E-mail address: hualin@sdu.edu.cn 